\title{
Uniting Low-Scale Leptogenesis Mechanisms
}

\author{
Juraj Klarić $\odot$, Mikhail Shaposhnikov, and Inar Timiryasov \\ Institute of Physics, Laboratory for Particle Physics and Cosmology, École polytechnique fédérale de Lausanne, \\ CH-1015 Lausanne, Switzerland
}

(Received 14 October 2020; revised 12 March 2021; accepted 6 July 2021; published 9 September 2021)

\begin{abstract}
In this Letter we demonstrate that what was previously considered as different mechanisms of baryon asymmetry generation involving two right-handed Majorana neutrinos with masses far below the Grand Unified Theory scale-leptogenesis via neutrino oscillations and resonant leptogenesis-are actually united. We show that the observed baryon asymmetry can be generated for all experimentally allowed values of the right-handed neutrino masses above $M_{N} \gtrsim 100 \mathrm{MeV}$. Leptogenesis is effective in a broad range of the parameters, including mass splitting between two right-handed neutrinos as big as $\Delta M_{N} / M_{N} \sim 0.1$, as well as mixing angles between the heavy and light neutrinos large enough to be accessible to planned intensity experiments or future colliders.
\end{abstract}

DOI: 10.1103/PhysRevLett.127.111802

Introduction.-Flavor oscillations of neutrinos are the only laboratory tested phenomenon pointing at the incompleteness of the standard model (SM). The presence of the ordinary baryonic matter in the observed amounts cannot be explained within the SM as well (see, e.g., review [1]). The minimal renormalizable extension of the SM contains two or more gauge singlet right-handed neutrinos which allow for a Dirac mass matrix $m_{D}$ for the neutrinos. These singlet right-handed neutrinos are the only particles which can have Majorana masses, with their mass matrix $M_{M}$. We do not know the values of these mass terms apriori; instead, they should be determined experimentally (like any other coefficients in front of renormalizable operators). Remarkably, diagonalizing the common neutrino mass matrix one finds that if $M_{M} \gg m_{D}$, the mass matrix of light neutrinos is $m_{\nu} \simeq-m_{D}^{2} / M_{M}$. This is the famous seesaw formula [2-7]. An important consequence of the theory is the mixing between the light and heavy neutrinos. This mixing allows the heavy neutrinos to interact with the rest of the SM, so from the experimental point of view they behave like heavy neutral leptons (HNLs). The search for HNLs is an important part of the physics programs of most existing accelerator experiments [8-14]. Planned high intensity frontier experiments [15-19], especially the Search for Hidden Particles (SHiP) experiment [20], provide an unparalleled opportunity if $M$ is in the region around a few $\mathrm{GeV}$, whereas future colliders, such as the Future Circular Collider in the lepton option (FCC-ee) [21-

Published by the American Physical Society under the terms of the Creative Commons Attribution 4.0 International license. Further distribution of this work must maintain attribution to the author(s) and the published article's title, journal citation, and DOI. Funded by SCOAP .
24], or the Circular Electron Positron Collider (CEPC) $[23,24]$ will cover a significant portion of the parameter space of heavier HNLs. The capability of explaining neutrino masses strongly motivates HNL searches. However, there are other intriguing consequences of the theory outlined above. Yukawa couplings of right-handed neutrinos can carry new sources of $\mathrm{CP}$ violation, while the HNLs themselves deviate from equilibrium in one way or another. Sphaleron processes in the early universe provide violation of the baryon number [25]. Therefore the Sakharov conditions can be satisfied and generation of the Baryon Asymmetry of the Universe (BAU) is possible. HNLs interact only with leptons, so it is the lepton asymmetry which is generated and transferred to the baryon sector by the sphaleron processes. This mechanism is known as leptogenesis. (Let us note in passing that transfer of asymmetry from the lepton sector is efficient at temperatures exceeding $\simeq 130 \mathrm{GeV}$ [26], this means that HNLs responsible for leptogenesis serve as a unique probe of the very early universe.) The suggestion along these lines was proposed by Fukugita and Yanagida [27] who considered very heavy right-handed neutrinos with masses above $10^{9} \mathrm{GeV}$ [28]. The mass scale of leptogenesis can be significantly lowered if two HNLs are nearly degenerate in mass; this phenomenon was dubbed resonant leptogenesis [29-39] (for more recent work see, e.g., [40-51]). It has also been realized that $\mathrm{GeV}$-scale right-handed neutrinos can also generate the BAU in leptogenesis via oscillations [52,53] (for more recent work see, e.g., [54-84]). In the minimal scenario with two heavy neutrinos, both leptogeneses require the HNLs to have nearly degenerate masses. The mass degeneracy of two HNLs is an interesting feature from the theoretical point of view as it may be a result of a global leptonic symmetry-in this case a pair of Majorana neutrinos $N$ can be joined into a quasi-Dirac 
fermion. This allows for sizable mixings between active neutrinos and HNLs in a technically natural way [85-94]. Note that in the case with three HNLs a mass degeneracy is no longer necessary for leptogenesis [61,81].

Resonant leptogenesis and leptogenesis via oscillations.-After inflation the baryon and lepton numbers of the universe as well as the number of HNLs may well be zero, and we will assume that this is indeed the case [95] (This is not necessarily so if the seesaw Lagrangian is supplemented by higher dimensional operators $[96,97]$.). The baryon asymmetry of the universe in both leptogeneses is produced in a set of processes including scatterings, decays, coherent oscillations of HNLs, and sphaleron transitions.

The conceptual difference between the two leptogeneses is the moment in the history of the universe when the asymmetry is generated. In resonant leptogenesis the BAU is usually generated when the temperature drops below the heavy neutrino mass, $T \lesssim M_{N}$, and the neutrinos begin to decay out of equilibrium $[38,39]$. As conversion between lepton and baryon number requires fast electroweak sphaleron processes, this implies a lower bound on the heavy neutrino masses around $M_{N} \sim T_{\text {sph }} \simeq 130 \mathrm{GeV}$ [26]. Indeed, this is close to the lowest heavy neutrino mass for which resonant leptogenesis was studied in [39].

On the other hand, in baryogenesis via neutrino oscillations, the BAU is primarily produced during the equilibration of the heavy neutrinos. It has been argued that baryogenesis via oscillation only works when $M_{N}$ is below $M_{W}$ [21]. For heavier HNLs decay channels into $W$ and $Z$ bosons open up, and a large equilibration rate wipes out deviation from equilibrium.

The question of whether the two leptogeneses-one at the $\mathrm{GeV}$ scale, and the other one above $M_{W}$-are indeed disconnected requires a systematic quantitative study. In this Letter we perform such a study using state-of-the-art rates and quantum kinetic equations for the first time. We show that the parameter spaces of the two leptogeneses are actually united, and that leptogenesis with two HNLs is operative for all values $M_{N}$ larger than a fraction of $\mathrm{GeV}$.

To avoid confusion with the terminology of oscillations and resonances (present in both mechanisms), in the remainder of the text, we borrow the language often used for dark matter production mechanisms, and refer to the two mechanisms as freeze-in leptogenesis, which mainly corresponds to leptogenesis via oscillations, where the BAU is mainly generated during the production of the HNLs, and freeze-out leptogenesis, which corresponds to conventional resonant leptogenesis, where the majority of the BAU is generated as the heavy neutrinos drop out of equilibrium due to the expansion of the universe.

A unified picture.-The first question one may ask when comparing the two mechanisms is whether the equations governing the production of the BAU are the same. There have been several approaches to deriving the evolution equations for resonant leptogenesis and leptogenesis via oscillations. In the case of resonant leptogenesis the perturbative computation leads to a divergent heavy neutrino decay asymmetry in the limit of exactly degenerate heavy neutrinos (see, e.g., [29]). This can be understood as a breakdown of the usual perturbation theory, since the unstable heavy neutrinos cannot appear as asymptotic $S$ matrix states. After the initial developments [29,31,33-37], the studies of resonant leptogenesis have taken a more formal turn with the goal of deriving the evolution equations from first principles, in particular using methods from nonequilibrium quantum field theory, in particular the closed-time-path (CTP) formalism [40-47,49,98-106]. For leptogenesis via neutrino oscillations, where the neutrinos are close to relativistic, the equations are often derived by generalizing the treatment of Sigl and Raffelt [107] of relativistic mixed neutrinos to the scenario with additional heavy states $[52,53]$. The same type of equations can be derived in the CTP formalism [45] if we assume a common mass shell for the two heavy neutrinos. This approach has successfully been used in studies of both resonant leptogenesis [48] and leptogenesis via neutrino oscillations [71], by taking the nonrelativistic and relativistic limits respectively. In this Letter we present, for the first time, a unified study accounting for all the effects with a single set of equations that leads to the two regimes of leptogenesis in the appropriate limits. The importance of nonrelativistic corrections to leptogenesis via oscillations was pointed out in $[50,75,76]$. The equations that we use in the remainder of this Letter are obtained from the ones used in $[75,80]$ after a change of basis (cf. also Refs. [83,108] and also $[45,71,78])$.

$$
\begin{aligned}
& i \frac{d n_{\Delta_{\alpha}}}{d t}=-2 i \frac{\mu_{\alpha}}{T} \int \frac{d^{3} k}{(2 \pi)^{3}} \operatorname{Tr}\left[\Gamma_{\alpha}\right] f_{N}\left(1-f_{N}\right) \\
&+i \int \frac{d^{3} k}{(2 \pi)^{3}} \operatorname{Tr}\left[\tilde{\Gamma}_{\alpha}\left(\bar{\rho}_{N}-\rho_{N}\right)\right], \\
& i \frac{d \rho_{N}}{d t}=\left[H_{N}, \rho_{N}\right]-\frac{i}{2}\left\{\Gamma, \rho_{N}-\rho_{N}^{\mathrm{eq}}\right\} \\
&-\frac{i}{2} \sum_{\alpha} \tilde{\Gamma}_{\alpha}\left[2 \frac{\mu_{\alpha}}{T} f_{N}\left(1-f_{N}\right)\right], \\
& i \frac{d \bar{\rho}_{N}}{d t}=-\left[H_{N}, \bar{\rho}_{N}\right]-\frac{i}{2}\left\{\Gamma, \bar{\rho}_{N}-\rho_{N}^{\mathrm{eq}}\right\} \\
&+\frac{i}{2} \sum_{\alpha} \tilde{\Gamma}_{\alpha}\left[2 \frac{\mu_{\alpha}}{T} f_{N}\left(1-f_{N}\right)\right],
\end{aligned}
$$

where $n_{\Delta_{\alpha}} \equiv L_{\alpha}-B / 3$ represent the comoving lepton asymmetries which can be related to the chemical potentials through the susceptibility matrix $\mu_{\beta}=\omega_{\alpha \beta} n_{\Delta_{\alpha}}$, and $\rho_{N}$ $\left(\bar{\rho}_{N}^{*}\right)$ is the matrix of the comoving number densities of HNLs of positive (negative) helicity. (Note that these 

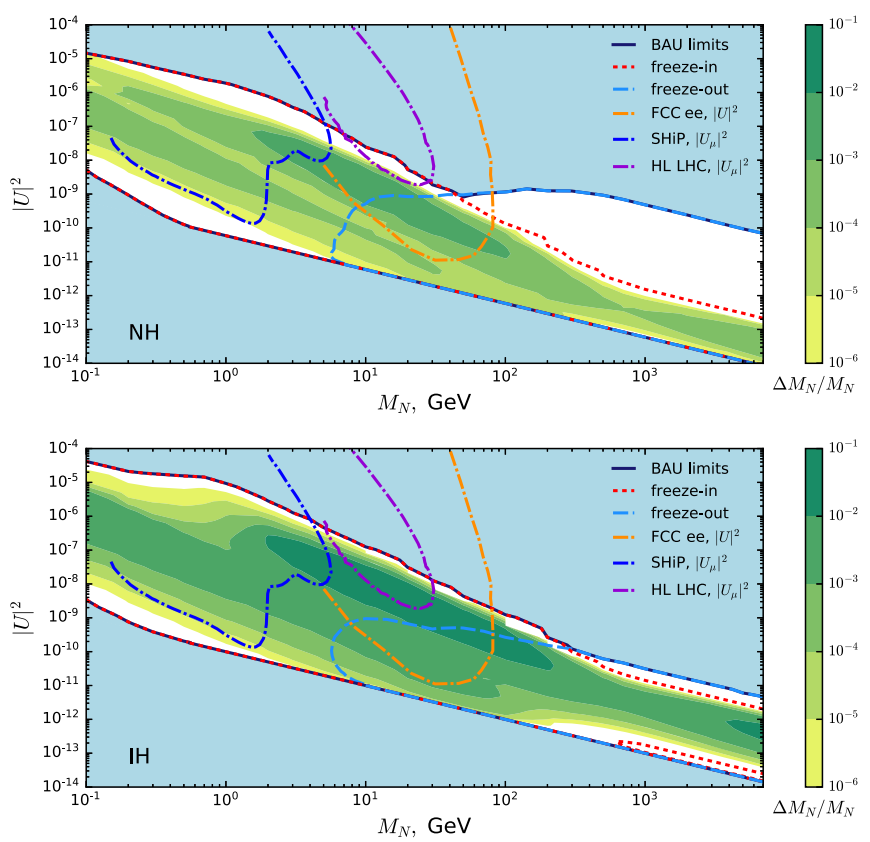

FIG. 1. Upper panel: normal hierarchy. Lower panel: inverted hierarchy. In the light blue region it is not possible to produce the observed BAU. Regions of parameter space corresponding to the freeze-in (no source term) and freeze-out regimes (thermal initial conditions) are bounded by the red dotted and light blue dashed lines, respectively. Together the two regimes span the whole lowscale leptogenesis parameter space. Freeze-in leptogenesis remains viable up to arbitrarily large masses, albeit for comparatively smaller mixing angles. The colored areas correspond to the maximal mass splitting consistent with leptogenesis for fixed $M$ and $U^{2}$. Note that the lower $|U|^{2}$ bound from leptogenesis coincides with the one imposed by the seesaw mechanism $|U|^{2} \geq$ $\sum_{i} m_{i} / M_{N}$ for $M_{N} \gtrsim 1 \mathrm{GeV}$. The white region corresponds to mass splittings below $10^{-6}$. One can see that the region of large mass splittings mostly coincides with the freeze-in leptogenesis regime. This can be expected, as for large mass splitting the majority of the BAU is generated at high temperatures before the HNLs begin to decay. For comparison we also show the expected sensitivities of SHiP (dark blue), high-luminosity large hadron collider (HL LHC) (purple), and FCC-ee (orange) as representative experiments in their corresponding mass range. The sensitivity lines are taken from [14,20,23].

equations need to be modified at $T>85 \mathrm{TeV}$, at which right-handed electrons are not in equilibrium.) The equations are governed by two sets of equilibration matrices $\Gamma=\sum_{\alpha} \Gamma_{\alpha}$ and $\tilde{\Gamma}=\sum_{\alpha} \tilde{\Gamma}_{\alpha}$ (the former describing the washout of asymmetries, whilst the latter quantifies the transfer of asymmetry between HNLs and leptons), the effective Hamiltonian $H_{N}$ (which describes the neutrino oscillations), and by the equilibrium matrix $\rho_{N}^{\mathrm{eq}}=\mathbf{1}_{2 \times 2} \cdot f_{N}+\mathcal{O}\left(\Delta M_{N} / E_{N}\right)$, where $f_{N}$ is the equilibrium distribution of the HNLs. Equation (1) describes both leptogeneses. Note that equations derived in Refs. [46,48] for the case of resonant leptogenesis have a similar form except for the fact that the equations for $\bar{\rho}_{N}$ are not independent from those for $\rho_{N}$ which is not the case in Eq. (1). However, there is no contradiction since in the nonrelativistic limit Eq. (1c) indeed becomes a conjugate of Eq. (1b). Another important distinction is that the equations from Ref. [46] contain the so-called effective Yukawa couplings [34,38]. We do not include such resummations in the present Letter, since a need for them does not arise in the derivations of quantum Boltzmann equations in either the Raffelt-Sigl $[52,53,76,108]$ or in the Wigner space CTP approaches $[45,71,78]$. The rates entering Eq. (1) pose the main theoretical challenge. A lot of effort has been made to compute them at high temperatures [57,59,109-112]; however, the rates in the literature are typically helicity averaged. For relativistic HNLs the rate is helicity dependent and requires a more careful calculation [67,76,79]. The helicity-dependent rates have only been calculated in the relativistic limit, and cannot be applied in the intermediate regime, which is crucial to connect the two mechanisms. In Ref. [113] we approximate the rate $\Gamma$ and show that the results are insensitive to the details of such estimates.

Parameter space of leptogenesis.-The system of equations (1) needs to be solved numerically to obtain an accurate estimate of the BAU. Solving momentumaveraged equations (see $[58,79]$ ), we perform a parameter scan over the masses and mixing angles consistent with the observed light neutrino masses using the Casas-Ibarra parametrization [114].

The neutrino flavor eigenstates can be expressed as $\nu_{\alpha}=U_{\alpha i} \nu_{i}+\Theta_{\alpha I} N_{I}^{c}$, where $\nu_{i}$ and $N_{I}$ are light and heavy mass eigenstates with masses $m_{i}$ and $M_{I}$ respectively, $U_{\alpha i}$ is the Pontecorvo-Maki-Nakagawa-Sakata (PMNS) matrix, and $\Theta_{\alpha I}$ is the mixing between active neutrinos and HNLs. Here we consider the case of two HNLs (The third HNLif it exists - could be light and very weakly coupled [89], which makes it a suitable dark matter candidate as is the case in the neutrino minimal standard model, $\nu$ MSM $[53,60,62,83,115]$.), which is compatible with the neutrino oscillation data, so $I=1,2$ and $M_{N_{1,2}}=M_{N} \pm \Delta M$. It is convenient to characterize the overall strength of the mixing using $|U|^{2}=\sum_{\alpha I}\left|\Theta_{\alpha I}\right|^{2}$. The seesaw requires that $|U|^{2} \geq \sum_{i} m_{i} / M_{N}$, whereas demanding successful leptogenesis sets up an upper bound on $|U|^{2}$ as well as a lower bound on $|U|^{2}$ for $M_{N} \lesssim 1 \mathrm{GeV}$. We do not consider the bound from Big Bang Nucleosynthesis [116-120]. In Fig. 1 we show the region in the parameter space where the observed value of the BAU can be generated. As one can see, the results depend on the neutrino mass hierarchy. One can show [113] that the allowed region extends to heavier masses and both upper and lower bounds scale as $|U|^{2} \propto 1 / M$. This scaling breaks down around $M \sim$ $10^{7} \mathrm{GeV}$ due to flavor effects [121-126], as well as the maximal mass splitting becoming of order $\Delta M / M \sim \mathcal{O}(1)$, which leads to a breakdown of the quasiparticle approximation used to derive the quantum kinetic equations. 
As one can see in Fig. 1, there is a continuous region in the $U^{2}-M$ plane where leptogenesis in its seemingly different incarnations is operative.

Regimes of leptogenesis. - As we can see from Fig. 1, there is no clear separation between the two leptogeneses. We distinguish between them based on when the majority of the asymmetry is generated, i.e., during freeze-in or freeze-out. To fully separate these regimes, we consider different initial conditions for the heavy neutrinos. For the freeze-out parameter space we start with thermalized heavy neutrinos, and rely purely on their deviation from equilibrium due to the expansion of the universe. This deviation is caused by the time derivative of the equilibrium distribution $\rho_{N}^{\mathrm{eq}}$, which we refer to as the source term. The presence of this source term can be seen when the equations are written in terms of the deviation of $\rho_{N}$ and $\bar{\rho}_{N}$ from equilibrium. Similarly, for freeze-in leptogenesis, we artificially turn off this source term and rely only on their vanishing initial abundance. The comparison between these three "parameter spaces" is shown in Fig. 1. The parameter space of freeze-out (freeze-in) leptogenesis is bounded by the light blue dashed (red dotted) line in Fig. 1. Of course, the physical solution relies on the presence of both effects and is bounded by the solid blue line in Fig. 1.

Perhaps surprisingly, we find that both regimes extend beyond the masses we would naively associate with them. Freeze-in leptogenesis extends far beyond $M_{W}$, and freezeout leptogenesis is possible already for masses as low as $\simeq 5 \mathrm{GeV}$. The main ingredients which make the overlap of these regimes possible are (i) flavor hierarchical washout, (ii) deviation from equilibrium due to the expansion of the universe, and (iii) approximate lepton number conservation.

When the heavy neutrino masses are of the same order as the temperature, the ratio of the HNL equilibration and Hubble rates is in general quite large, with the smallest value for normal hierarchy being around $\mathcal{O}(30)$. Naively this would lead us to expect that any asymmetries generated during freeze-in would be erased by the strong washout. However, the washout rate of a particular lepton flavor can be several orders of magnitude smaller than the equilibration rate for the heavy neutrinos. The presence of a flavor hierarchical washout is almost completely determined by the CP-violating phase $\delta$ and the Majorana phases from the PMNS matrix as parametrized in [127]. It can range from $\mathcal{O}\left(10^{-3}\right)$ to $\mathcal{O}\left(10^{-1}\right)$ for normal hierarchy, while it can be as small as $\mathcal{O}\left(10^{-4}\right)$, or completely nonhierarchical in the case of inverted hierarchy. For large masses of HNLs, freeze-in leptogenesis crucially depends on the presence of such hierarchies (cf. [128], where the importance of a hierarchical washout was pointed out in the $3 \mathrm{HNL}$ case). Furthermore, we find that freeze-in is the dominant mechanism when the mass splitting between the heavy neutrinos is sizable $\Delta M_{N} / M_{N} \sim \mathcal{O}\left(10^{-2}\right)$, as demonstrated in Fig. 1.
At the same time, we find successful freeze-out leptogenesis at the few $\mathrm{GeV}$ scale. The main reason behind this effect is that the decay asymmetries of the heavy neutrinos can be close to $\mathcal{O}(1)$. The deviation from equilibrium caused by the heavy neutrino freeze-out in such a scenario will be suppressed by $10^{-3} M^{2} / T^{2}$, and can still lead to the observed baryon asymmetry.

Finally, we also find that even in the absence of flavor hierarchical washout, large mixing angles remain viable for heavy neutrino masses above $M_{W}$. The main reason behind this observation is the presence of an approximately conserved lepton number. If the pair of heavy Majorana neutrinos is close to degenerate in mass, they can be combined into a single pseudo-Dirac neutrino which can carry a lepton number. This type of scenario was studied as a technically natural way of adding light right-handed neutrinos to the SM $[85-92,129]$. However, the importance of an approximate lepton number in preventing large washout during leptogenesis was first noted in [130]. The small parameter determining the conservation of this lepton number is the ratio of the heavy neutrino mass splitting and their interaction (decay) rate.

Resonant leptogenesis with $\mathrm{GeV}$-scale heavy neutrinos was considered in Ref. [50]. (Recently a similar study was also performed in [51], with a different calculation of the rates for the $1 \leftrightarrow 2$ processes. Another similar study was performed in [131], with an emphasis on the importance of the lepton flavor violating decay asymmetry.) In this Letter it was suggested that the parameter space of resonant leptogenesis could extend to $\mathrm{GeV}$-scale heavy neutrino masses. Our results confirm this estimate and establish a similar but not identical lower bound. The analysis of Ref. [50] was based on qualitative arguments relying on Boltzmann equations, and it did not take into account the full time dependence of the decay asymmetry. In fact, it is known that a decay asymmetry provides an accurate description of leptogenesis only when certain conditions are met-when the mass splitting between heavy neutrinos is much larger than the equilibration rate $\Gamma$, or when all the eigenvalues of the heavy neutrino equilibration matrix $\Gamma$ are much bigger than the Hubble rate $H[45,46,48,132]$. Neither of these conditions is fulfilled in Refs. [50,68,131]. Moreover, flavor effects which are crucial for freeze-in leptogenesis were neglected; these effects were included in a more recent study [131]. The refined study using the density-matrix formalism from [68] shows semiquantitative agreement in the mass range of a few $\mathrm{GeV}$, but also indicates that the use of Boltzmann equations may lead to a significant overestimate of the produced BAU for larger heavy neutrino masses (up to $10 \mathrm{GeV}$, where their study of the parameter space halts). Furthermore, the processes giving the dominant contributions to the rates have been neglected (see, e.g., discussion in [80]) in Refs. $[50,68]$. In our study we do not rely on these approximations and do not limit the mass splitting to specific values, as was done in $[50,68]$. Note that in [113] we 
compare the Boltzmann and density-matrix approaches, and show how the former arises in the appropriate limits of the latter. As a consequence, we find qualitatively very different limits on $|U|^{2}$ and $\Delta M_{N} / M_{N}$.

Discussion and conclusions.-In this Letter we investigate the similarities and differences between resonant leptogenesis and leptogenesis via neutrino oscillations in the minimal extension of the standard model by two HNLs. The two mechanisms are closely related, and the equations needed to describe them are in fact the same. Since the defining feature of resonant leptogenesis, namely the resonant production of the baryon asymmetry, is also present in leptogenesis via neutrino oscillations, we focus on the major difference between the two mechanisms, namely the question of whether the majority of the BAU is produced during the freeze-in, or freeze-out of the heavy neutrinos.

We found significant overlap between the two regimes, namely, freeze-in leptogenesis turns out to play a major role in generating the $\mathrm{BAU}$ even for $\mathrm{TeV}$ and heavier Majorana neutrinos. This regime mainly coincides with relatively large $\Delta M_{N} / M_{N} \sim \mathcal{O}\left(10^{-2}\right)$ mass splitting, compared with the one optimal for a resonant enhancement $\Delta M_{N}$ / $M_{N} \sim 10^{-11}$. Furthermore, the fact that the freeze-in regime extends to large masses implies a strong dependence on the initial condition which was typically absent in resonant leptogenesis.

On the other hand, we also find viable realizations of freeze-out leptogenesis for masses as low as $M \simeq 5 \mathrm{GeV}$. This can be understood through the large decay efficiency of the HNLs, as a suppression factor of $M^{2} / T^{2} \sim 10^{-3}$ is not sufficiently small to prevent baryogenesis.

Together, these two parametric regimes span all experimentally allowed masses for the heavy neutrinos, from a fraction of $\mathrm{GeV}$, to $M_{W}$, and beyond.

The absence of a preferred mass scale of leptogenesis calls for a vast and diverse search program.

We thank Marco Drewes, Shintaro Eijima, Björn Garbrecht, Jacopo Ghiglieri, Mikko Laine, Apostolos Pilaftsis, and Daniele Teresi for helpful comments and discussions. This work was supported by ERC-AdG-2015 Grant No. 694896 and by the Swiss National Science Foundation Excellence, Grant No. 200020B_182864.

[1] L. Canetti, M. Drewes, and M. Shaposhnikov, Matter and antimatter in the Universe, New J. Phys. 14, 095012 (2012).

[2] P. Minkowski, $\mu \rightarrow e \gamma$ at a rate of one out of $10^{9}$ muon decays?, Phys. Lett. 67B, 421 (1977).

[3] T. Yanagida, Horizontal gauge symmetry and masses of neutrinos, Prog. Theor. Phys., 64, 1103 (1980).

[4] M. Gell-Mann, P. Ramond, and R. Slansky, Complex spinors and unified theories, Conf. Proc. C 790927, 315 (1979).
[5] R. N. Mohapatra and G. Senjanovic, Neutrino Mass and Spontaneous Parity Nonconservation, Phys. Rev. Lett. 44, 912 (1980).

[6] J. Schechter and J. W. F. Valle, Neutrino masses in $\mathrm{SU}(2) \times \mathrm{U}(1)$ theories, Phys. Rev. D 22, 2227 (1980).

[7] J. Schechter and J. W. F. Valle, Neutrino decay and spontaneous violation of lepton number, Phys. Rev. D 25, 774 (1982).

[8] D. Liventsev et al. (Belle Collaboration), Search for heavy neutrinos at Belle, Phys. Rev. D 87, 071102 (2013); Erratum, Phys. Rev. D 95, 099903 (2017).

[9] R. Aaij et al. (LHCb Collaboration), Search for Majorana Neutrinos in $B^{-} \rightarrow \pi^{+} \mu^{-} \mu^{-}$Decays, Phys. Rev. Lett. 112, 131802 (2014).

[10] A. V. Artamonov et al. (E949 Collaboration), Search for heavy neutrinos in $K^{+} \rightarrow \mu^{+} \nu_{H}$ decays, Phys. Rev. D 91, 052001 (2015); Erratum, Phys. Rev. D 91, 059903 (2015).

[11] G. Aad et al. (ATLAS Collaboration), Search for heavy Majorana neutrinos with the ATLAS detector in $p p$ collisions at $\sqrt{s}=8 \mathrm{TeV}$, J. High Energy Phys. 07 (2015) 162.

[12] V. Khachatryan et al. (CMS Collaboration), Search for heavy Majorana neutrinos in $\mu^{ \pm} \mu^{ \pm}+$jets events in protonproton collisions at $\sqrt{s}=8 \mathrm{TeV}$, Phys. Lett. B 748, 144 (2015).

[13] A. M. Sirunyan et al. (CMS Collaboration), Search for Heavy Neutral Leptons in Events with Three Charged Leptons in Proton-Proton Collisions at $\sqrt{s}=13 \mathrm{TeV}$, Phys. Rev. Lett. 120, 221801 (2018).

[14] I. Boiarska, K. Bondarenko, A. Boyarsky, S. Eijima, M. Ovchynnikov, O. Ruchayskiy, and I. Timiryasov, Probing baryon asymmetry of the Universe at LHC and SHiP, arXiv:1902.04535.

[15] V. V. Gligorov, S. Knapen, M. Papucci, and D. J. Robinson, Searching for long-lived particles: A compact detector for exotics at LHCb, Phys. Rev. D 97, 015023 (2018).

[16] J. L. Feng, I. Galon, F. Kling, and S. Trojanowski, Forward search experiment at the LHC, Phys. Rev. D 97, 035001 (2018).

[17] F. Kling and S. Trojanowski, Heavy neutral leptons at FASER, Phys. Rev. D 97, 095016 (2018).

[18] M. Drewes, J. Hajer, J. Klaric, and G. Lanfranchi, NA62 sensitivity to heavy neutral leptons in the low scale seesaw model, J. High Energy Phys. 07 (2018) 105.

[19] R. K. Ellis et al., Physics briefing book: Input for the european strategy for particle physics update 2020, arXiv:1910.11775.

[20] S. Alekhin et al., A facility to Search for Hidden Particles at the CERN SPS: The SHiP physics case, Rep. Prog. Phys. 79, 124201 (2016).

[21] A. Blondel, E. Graverini, N. Serra, and M. Shaposhnikov (FCC-ee study Team Collaboration), Search for heavy right handed neutrinos at the FCC-ee, Nucl. Part. Phys. Proc. 273-275, 1883 (2016).

[22] A. Abada et al. (FCC Collaboration), FCC physics opportunities, Eur. Phys. J. C 79, 474 (2019).

[23] S. Antusch, E. Cazzato, and O. Fischer, Sterile neutrino searches at future $e^{-} e^{+}, p p$, and $e^{-} p$ colliders, Int. J. Mod. Phys. A 32, 1750078 (2017). 
[24] S. Antusch, E. Cazzato, and O. Fischer, Displaced vertex searches for sterile neutrinos at future lepton colliders, J. High Energy Phys. 12 (2016) 007.

[25] V. A. Kuzmin, V. A. Rubakov, and M. E. Shaposhnikov, On the anomalous electroweak baryon number nonconservation in the early Universe, Phys. Lett. 155B, 36 (1985).

[26] M. D’Onofrio, K. Rummukainen, and A. Tranberg, Sphaleron Rate in the Minimal Standard Model, Phys. Rev. Lett. 113, 141602 (2014).

[27] M. Fukugita and T. Yanagida, Baryogenesis without grand unification, Phys. Lett. B 174, 45 (1986).

[28] S. Davidson and A. Ibarra, A lower bound on the righthanded neutrino mass from leptogenesis, Phys. Lett. B 535, 25 (2002).

[29] J. Liu and G. Segre, Reexamination of generation of baryon and lepton number asymmetries by heavy particle decay, Phys. Rev. D 48, 4609 (1993).

[30] M. Flanz, E. A. Paschos, and U. Sarkar, Baryogenesis from a lepton asymmetric universe, Phys. Lett. B 345, 248 (1995); Erratum, Phys. Lett. B 384, 487 (1996); Erratum, Phys. Lett. B 382, 447 (1996).

[31] M. Flanz, E. A. Paschos, U. Sarkar, and J. Weiss, Baryogenesis through mixing of heavy Majorana neutrinos, Phys. Lett. B 389, 693 (1996).

[32] L. Covi, E. Roulet, and F. Vissani, CP violating decays in leptogenesis scenarios, Phys. Lett. B 384, 169 (1996).

[33] L. Covi and E. Roulet, Baryogenesis from mixed particle decays, Phys. Lett. B 399, 113 (1997).

[34] A. Pilaftsis, CP violation and baryogenesis due to heavy Majorana neutrinos, Phys. Rev. D 56, 5431 (1997).

[35] A. Pilaftsis, Resonant CP violation induced by particle mixing in transition amplitudes, Nucl. Phys. B504, 61 (1997).

[36] A. Pilaftsis, Heavy Majorana neutrinos and baryogenesis, Int. J. Mod. Phys. A 14, 1811 (1999).

[37] W. Buchmuller and M. Plumacher, CP asymmetry in Majorana neutrino decays, Phys. Lett. B 431, 354 (1998).

[38] A. Pilaftsis and Thomas E. J. Underwood, Resonant leptogenesis, Nucl. Phys. B692, 303 (2004).

[39] A. Pilaftsis and Thomas E. J. Underwood, Electroweakscale resonant leptogenesis, Phys. Rev. D 72, 113001 (2005).

[40] A. De Simone and A. Riotto, On resonant leptogenesis, J. Cosmol. Astropart. Phys. 08 (2007) 013.

[41] A. De Simone and A. Riotto, Quantum Boltzmann equations and leptogenesis, J. Cosmol. Astropart. Phys. 08 (2007) 002.

[42] M. Garny, A. Hohenegger, A. Kartavtsev, and M. Lindner, Systematic approach to leptogenesis in nonequilibrium QFT: Self-energy contribution to the CP-violating parameter, Phys. Rev. D 81, 085027 (2010).

[43] M. Garny, A. Kartavtsev, and A. Hohenegger, Leptogenesis from first principles in the resonant regime, Ann. Phys. (Amsterdam) 328, 26 (2013).

[44] S. Iso, K. Shimada, and M. Yamanaka, Kadanoff-Baym approach to the thermal resonant leptogenesis, J. High Energy Phys. 04 (2014) 062.

[45] B. Garbrecht and M. Herranen, Effective theory of resonant leptogenesis in the closed-time-path approach, Nucl. Phys. B861, 17 (2012).
[46] P. S. Bhupal Dev, P. Millington, A. Pilaftsis, and D. Teresi, Flavour covariant transport equations: An application to resonant leptogenesis, Nucl. Phys. B886, 569 (2014).

[47] P. S. Bhupal Dev, P. Millington, A. Pilaftsis, and D. Teresi, Flavour covariant formalism for resonant leptogenesis, Nucl. Part. Phys. Proc. 273-275, 268 (2016).

[48] B. Garbrecht, F. Gautier, and J. Klaric, Strong washout approximation to resonant leptogenesis, J. Cosmol. Astropart. Phys. 09 (2014) 033.

[49] P. S. Bhupal Dev, P. Millington, A. Pilaftsis, and D. Teresi, Corrigendum to "flavour covariant transport equations: an application to resonant leptogenesis", Nucl. Phys. B897, 749 (2015).

[50] T. Hambye and D. Teresi, Higgs Doublet Decay as the Origin of the Baryon Asymmetry, Phys. Rev. Lett. 117, 091801 (2016).

[51] X.-M. Jiang, Y.-L. Tang, Z.-H. Yu, and H.-H. Zhang, $1 \leftrightarrow$ 2 Processes of a sterile neutrino around electroweak scale in the thermal plasma, Phys. Rev. D 103, 095003 (2021).

[52] E. K. Akhmedov, V. A. Rubakov, and A. Yu. Smirnov, Baryogenesis via Neutrino Oscillations, Phys. Rev. Lett. 81, 1359 (1998).

[53] T. Asaka and M. Shaposhnikov, The $\nu$ MSM, dark matter and baryon asymmetry of the universe, Phys. Lett. B 620 , 17 (2005).

[54] M. Shaposhnikov, The $\nu$ MSM, leptonic asymmetries, and properties of singlet fermions, J. High Energy Phys. 08 (2008) 008.

[55] L. Canetti and M. Shaposhnikov, Baryon asymmetry of the Universe in the $\nu$ MSM, J. Cosmol. Astropart. Phys. 09 (2010) 001.

[56] T. Asaka and H. Ishida, Flavour mixing of neutrinos and baryon asymmetry of the Universe, Phys. Lett. B 692, 105 (2010).

[57] A. Anisimov, D. Besak, and D. Bodeker, Thermal production of relativistic Majorana neutrinos: Strong enhancement by multiple soft scattering, J. Cosmol. Astropart. Phys. 03 (2011) 042.

[58] T. Asaka, S. Eijima, and H. Ishida, Kinetic equations for baryogenesis via sterile neutrino oscillation, J. Cosmol. Astropart. Phys. 02 (2012) 021.

[59] D. Besak and D. Bodeker, Thermal production of ultrarelativistic right-handed neutrinos: Complete leading-order results, J. Cosmol. Astropart. Phys. 03 (2012) 029.

[60] L. Canetti, M. Drewes, and M. Shaposhnikov, Sterile Neutrinos as the Origin of Dark and Baryonic Matter, Phys. Rev. Lett. 110, 061801 (2013).

[61] M. Drewes and B. Garbrecht, Leptogenesis from a GeV seesaw without mass degeneracy, J. High Energy Phys. 03 (2013) 096.

[62] L. Canetti, M. Drewes, T. Frossard, and M. Shaposhnikov, Dark Matter, baryogenesis and neutrino oscillations from right handed neutrinos, Phys. Rev. D 87, 093006 (2013).

[63] B. Shuve and I. Yavin, Baryogenesis through neutrino oscillations: A unified perspective, Phys. Rev. D 89, 075014 (2014).

[64] D. Bodeker and M. Laine, Kubo relations and radiative corrections for lepton number washout, J. Cosmol. Astropart. Phys. 05 (2014) 041. 
[65] A. Abada, G. Arcadi, V. Domcke, and M. Lucente, Lepton number violation as a key to low-scale leptogenesis, J. Cosmol. Astropart. Phys. 11 (2015) 041.

[66] P. Hernández, M. Kekic, J. López-Pavón, J. Racker, and N. Rius, Leptogenesis in GeV scale seesaw models, J. High Energy Phys. 10 (2015) 067.

[67] J. Ghiglieri and M. Laine, Neutrino dynamics below the electroweak crossover, J. Cosmol. Astropart. Phys. 07 (2016) 015.

[68] T. Hambye and D. Teresi, Baryogenesis from L-violating Higgs-doublet decay in the density-matrix formalism, Phys. Rev. D 96, 015031 (2017).

[69] M. Drewes and S. Eijima, Neutrinoless double $\beta$ decay and low scale leptogenesis, Phys. Lett. B 763, 72 (2016).

[70] T. Asaka, S. Eijima, and H. Ishida, On neutrinoless double beta decay in the $\nu$ MSM, Phys. Lett. B 762, 371 (2016).

[71] M. Drewes, B. Garbrecht, D. Gueter, and J. Klaric, Leptogenesis from oscillations of heavy neutrinos with large mixing angles, J. High Energy Phys. 12 (2016) 150.

[72] P. Hernández, M. Kekic, J. López-Pavón, J. Racker, and J. Salvado, Testable baryogenesis in seesaw models, J. High Energy Phys. 08 (2016) 157.

[73] M. Drewes, B. Garbrecht, D. Gueter, and J. Klaric, Testing the low scale seesaw and leptogenesis, J. High Energy Phys. 08 (2017) 018.

[74] T. Asaka, S. Eijima, H. Ishida, K. Minogawa, and T. Yoshii, Initial condition for baryogenesis via neutrino oscillation, Phys. Rev. D 96, 083010 (2017).

[75] S. Eijima and M. Shaposhnikov, Fermion number violating effects in low scale leptogenesis, Phys. Lett. B 771, 288 (2017).

[76] J. Ghiglieri and M. Laine, GeV-scale hot sterile neutrino oscillations: A derivation of evolution equations, J. High Energy Phys. 05 (2017) 132.

[77] S. Eijima, M. Shaposhnikov, and I. Timiryasov, Freeze-out of baryon number in low-scale leptogenesis, J. Cosmol. Astropart. Phys. 11 (2017) 030.

[78] S. Antusch, E. Cazzato, M. Drewes, O. Fischer, B. Garbrecht, D. Gueter, and J. Klaric, Probing leptogenesis at future colliders, J. High Energy Phys. 09 (2018) 124.

[79] J. Ghiglieri and M. Laine, GeV-scale hot sterile neutrino oscillations: A numerical solution, J. High Energy Phys. 02 (2018) 078.

[80] S. Eijima, M. Shaposhnikov, and I. Timiryasov, Parameter space of baryogenesis in the $\nu$ MSM, J. High Energy Phys. 07 (2019) 077.

[81] A. Abada, G. Arcadi, V. Domcke, M. Drewes, J. Klaric, and M. Lucente, Low-scale leptogenesis with three heavy neutrinos, J. High Energy Phys. 01 (2019) 164.

[82] J. Ghiglieri and M. Laine, Precision study of GeV-scale resonant leptogenesis, J. High Energy Phys. 02 (2019) 014.

[83] J. Ghiglieri and M. Laine, Sterile neutrino dark matter via GeV-scale leptogenesis? J. High Energy Phys. 07 (2019) 078.

[84] J. Ghiglieri and M. Laine, Sterile neutrino dark matter via coinciding resonances, J. Cosmol. Astropart. Phys. 07 (2020) 012.

[85] D. Wyler and L. Wolfenstein, Massless neutrinos in leftright symmetric models, Nucl. Phys. B218, 205 (1983).
[86] R. N. Mohapatra and J. W. F. Valle, Neutrino mass and baryon number nonconservation in superstring models, Phys. Rev. D 34, 1642 (1986).

[87] G. C. Branco, W. Grimus, and L. Lavoura, The seesaw mechanism in the presence of a conserved lepton number, Nucl. Phys. B312, 492 (1989).

[88] M. C. Gonzalez-Garcia and J. W. F. Valle, Fast decaying neutrinos and observable flavor violation in a new class of majoron models, Phys. Lett. B 216, 360 (1989).

[89] M. Shaposhnikov, A Possible symmetry of the $\nu \mathrm{MSM}$, Nucl. Phys. B763, 49 (2007).

[90] J. Kersten and A. Yu. Smirnov, Right-handed neutrinos at CERN LHC and the mechanism of neutrino mass generation, Phys. Rev. D 76, 073005 (2007).

[91] A. Abada, C. Biggio, F. Bonnet, M. B. Gavela, and T. Hambye, Low energy effects of neutrino masses, J. High Energy Phys. 12 (2007) 061.

[92] M. B. Gavela, T. Hambye, D. Hernandez, and P. Hernandez, Minimal flavour seesaw models, J. High Energy Phys. 09 (2009) 038.

[93] M. Drewes, J. Klarić, and P. Klose, On lepton number violation in heavy neutrino decays at colliders, J. High Energy Phys. 11 (2019) 032.

[94] C. N. Leung and S. T. Petcov, A comment on the coexistence of Dirac and Majorana massive neutrinos, Phys. Lett. 125B, 461 (1983).

[95] F. Bezrukov, D. Gorbunov, and M. Shaposhnikov, On initial conditions for the Hot Big Bang, J. Cosmol. Astropart. Phys. 06 (2009) 029.

[96] F. Bezrukov, D. Gorbunov, and M. Shaposhnikov, Late and early time phenomenology of Higgs-dependent cutoff, J. Cosmol. Astropart. Phys. 10 (2011) 001.

[97] M. Shaposhnikov, A. Shkerin, I. Timiryasov, and S. Zell, Einstein-Cartan Portal to Dark Matter, Phys. Rev. Lett. 126, 161301 (2021).

[98] W. Buchmuller and S. Fredenhagen, Quantum mechanics of baryogenesis, Phys. Lett. B 483, 217 (2000).

[99] M. Garny, A. Hohenegger, A. Kartavtsev, and M. Lindner, Systematic approach to leptogenesis in nonequilibrium QFT: Vertex contribution to the CP-violating parameter, Phys. Rev. D 80, 125027 (2009).

[100] A. Anisimov, W. Buchmüller, M. Drewes, and S. Mendizabal, Leptogenesis from Quantum Interference in a Thermal Bath, Phys. Rev. Lett. 104, 121102 (2010).

[101] M. Beneke, B. Garbrecht, M. Herranen, and P. Schwaller, Finite number density corrections to leptogenesis, Nucl. Phys. B838, 1 (2010).

[102] M. Herranen, K. Kainulainen, and P. M. Rahkila, Coherent quantum Boltzmann equations from cQPA, J. High Energy Phys. 12 (2010) 072.

[103] C. Fidler, M. Herranen, K. Kainulainen, and P. M. Rahkila, Flavoured quantum Boltzmann equations from cQPA, J. High Energy Phys. 02 (2012) 065.

[104] M. Herranen, K. Kainulainen, and P. M. Rahkila, Flavourcoherent propagators and Feynman rules: Covariant cQPA formulation, J. High Energy Phys. 02 (2012) 080.

[105] P. Millington and A. Pilaftsis, Perturbative nonequilibrium thermal field theory, Phys. Rev. D 88, 085009 (2013). 
[106] P. Millington and A. Pilaftsis, Perturbative nonequilibrium thermal field theory to all orders in gradient expansion, Phys. Lett. B 724, 56 (2013).

[107] G. Sigl and G. Raffelt, General kinetic description of relativistic mixed neutrinos, Nucl. Phys. B406, 423 (1993).

[108] D. Bödeker and D. Schröder, Kinetic equations for sterile neutrinos from thermal fluctuations, J. Cosmol. Astropart. Phys. 02 (2020) 033.

[109] I. Ghisoiu and M. Laine, Right-handed neutrino production rate at $T>160 \mathrm{GeV}$, J. Cosmol. Astropart. Phys. 12 (2014) 032.

[110] B. Garbrecht, F. Glowna, and P. Schwaller, Scattering rates for leptogenesis: Damping of lepton flavour coherence and production of singlet neutrinos, Nucl. Phys. B877, 1 (2013).

[111] S. Biondini et al., Status of rates and rate equations for thermal leptogenesis, Int. J. Mod. Phys. A 33, 1842004 (2018).

[112] B. Garbrecht, P. Klose, and C. Tamarit, Relativistic and spectator effects in leptogenesis with heavy sterile neutrinos, J. High Energy Phys. 02 (2020) 117.

[113] J. Klaric, M. Shaposhnikov, and I. Timiryasov, Reconciling resonant and low-scale leptogenesis (to be published).

[114] J. A. Casas and A. Ibarra, Oscillating neutrinos and $\mu \rightarrow$ e $\gamma$, Nucl. Phys. B618, 171 (2001).

[115] T. Asaka, S. Blanchet, and M. Shaposhnikov, The $\nu$ MSM, dark matter and neutrino masses, Phys. Lett. B 631, 151 (2005).

[116] A. D. Dolgov, S. H. Hansen, G. Raffelt, and D. V. Semikoz, Heavy sterile neutrinos: Bounds from big bang nucleosynthesis and Sn1987A, Nucl. Phys. B590, 562 (2000).

[117] O. Ruchayskiy and A. Ivashko, Restrictions on the lifetime of sterile neutrinos from primordial nucleosynthesis, J. Cosmol. Astropart. Phys. 10 (2012) 014.

[118] G. B. Gelmini, M. Kawasaki, A. Kusenko, K. Murai, and V. Takhistov, Big bang nucleosynthesis constraints on sterile neutrino and lepton asymmetry of the Universe, J. Cosmol. Astropart. Phys. 09 (2020) 051.
[119] N. Sabti, A. Magalich, and A. Filimonova, An extended analysis of heavy neutral leptons during big bang nucleosynthesis, J. Cosmol. Astropart. Phys. 11 (2020) 056.

[120] A. Boyarsky, M. Ovchynnikov, O. Ruchayskiy, and V. Syvolap, Improved BBN constraints on heavy neutral leptons, Phys. Rev. D 104, 023517 (2021).

[121] A. Abada, S. Davidson, F.-X. Josse-Michaux, M. Losada, and A. Riotto, Flavor issues in leptogenesis, J. Cosmol. Astropart. Phys. 04 (2006) 004.

[122] E. Nardi, Y. Nir, E. Roulet, and J. Racker, The importance of flavor in leptogenesis, J. High Energy Phys. 01 (2006) 164.

[123] A. Abada, S. Davidson, A. Ibarra, F. X. Josse-Michaux, M. Losada, and A. Riotto, Flavour matters in leptogenesis, J. High Energy Phys. 09 (2006) 010.

[124] S. Blanchet and P. Di Bari, Flavor effects on leptogenesis predictions, J. Cosmol. Astropart. Phys. 03 (2007) 018.

[125] S. Pascoli, S. T. Petcov, and A. Riotto, Connecting low energy leptonic CP-violation to leptogenesis, Phys. Rev. D 75, 083511 (2007).

[126] A. De Simone and A. Riotto, On the impact of flavour oscillations in leptogenesis, J. Cosmol. Astropart. Phys. 02 (2007) 005.

[127] P. A. Zyla et al. (Particle Data Group Collaboration), Review of particle physics, Prog. Theor. Exp. Phys. 2020, 083C01 (2020).

[128] B. Garbrecht, More viable parameter space for leptogenesis, Phys. Rev. D 90, 063522 (2014).

[129] C. N.Leung and S. T.Petcov, A comment on the coexistence of Dirac and Majorana massive neutrinos, Phys. Lett. 125B, 461 (1983).

[130] S. Blanchet, T. Hambye, and F.-X. Josse-Michaux, Reconciling leptogenesis with observable $\mu \rightarrow$ er rates, J. High Energy Phys. 04 (2010) 023.

[131] A. Granelli, K. Moffat, and S. T. Petcov, Flavoured resonant leptogenesis at Sub-TeV scales, arXiv: 2009.03166.

[132] S. Iso and K. Shimada, Coherent flavour oscillation and $\mathrm{CP}$ violating parameter in thermal resonant leptogenesis, J. High Energy Phys. 08 (2014) 043. 